\title{
A Case Report of Surgical Resections with Local and Systemic Chemotherapy for Three Recurrences of Colon Cancer Occurring Ten Years after Colectomy
}

\author{
Hisanori Miki, d Kozo Tsunemi ${ }^{a}$ Masao Toyoda ${ }^{a}$ \\ Hideto Senzaki $^{b}$ Yutaka Yonemura $^{c}$ Airo Tsubura ${ }^{d}$ \\ Divisions of ${ }^{\mathrm{a}}$ Surgery and ${ }^{\mathrm{b}}$ Pathology, Osaka Saiseikai Nakatsu Hospital, Osaka, \\ 'Division of Surgery, Kishiwada Tokushukai Hospital, Kishiwada, and \\ ${ }^{\mathrm{d}}$ Department of Pathology II, Kansai Medical University, Moriguchi, Japan
}

\section{Key Words}

Colon cancer - Dormancy - Intra-abdominal recurrence - Surgical resection . Intraperitoneal chemotherapy · Vascular endothelial growth factor (VEGF) · CD44

\begin{abstract}
A 56-year-old Japanese woman who underwent a curative resection of ascending colon cancer at 43 years of age was found to have a tumor in her lower left abdominal cavity by computed tomography at 53 years of age. The tumor in the omentum was resected and identified as an adenocarcinoma compatible with metastasis from the primary ascending colon cancer. Although the patient received adjuvant chemotherapy with tegafur uracil and calcium folinate, liver metastasis was detected 9 months after the first recurrence. A segmentectomy and hepatectomy was performed, and histopathological findings indicated metastasis from the primary colon cancer. A third recurrence was detected in the right abdominal cavity 7 months after the second surgery. The patient received 5 cycles of combination chemotherapy consisting of folinic acid, fluorouracil and irinotecan before the third operation. The metastatic tumor resection together with intraperitoneal chemotherapy was performed, and histopathological findings indicated metastasis from the primary colon cancer. After the third surgery, the patient received adjuvant chemotherapy consisting of 5 cycles of folinic acid, fluorouracil and oxaliplatin. The patient is well with no evidence of recurrence 12 months after the third recurrence. This case suggests that colon cancer can be dormant for over 10 years and that long-term follow-up is required after curative resection.
\end{abstract}


Aggressive local as well as systemic chemotherapy may be required for the management of colon cancer recurrence.

\section{Introduction}

Colon cancer is the most common form of cancer worldwide and the second leading cause of cancer-related death in the Western world [1]. Survival is related to stage, and the 5 -year overall survival rate is approximately $60 \%$. However, advanced stages with metastasis show a 5-year survival rate of only 5\% [2]. Metachronous secondary tumors affect $20-30 \%$ of colon cancer patients and are usually detected within 2 years after the resection of the primary tumor [3]. However, there is still no consensus concerning the therapy and the duration of follow-up of patients who have had curative colonic resection. Recently, peritonectomy combined with intraperitoneal chemotherapy was reported to improve the long-term survival of peritoneal carcinomatosis [4-6], but the role of intraperitoneal chemotherapy in addition to adjuvant chemotherapy and tumor resection remains undefined in patients with intra-abdominal recurrence of colon cancer. We herein report a case of ascending colon cancer with intraperitoneal recurrence 10 years after the resection of the primary lesion. Treatment consisted of three resections against the intra-abdominal metastatic lesion and systemic and localized (intraperitoneal) chemotherapy, which resulted in no evidence of a fourth recurrence 12 months after the third recurrence.

\section{Case Report}

A 56-year-old Japanese woman who had a history of ascending colon cancer underwent radical right hemicolectomy with D3 lymph node dissection at 43 years of age (fig. 1). The pathological findings were pSS (moderately differentiated, ly1, v1, INFb) pN1, sH0, sP0, fStage IIIa, Cur A, and the case was classified as T3N1M0 (stage IIIa) according to the TNM classification. The patient did not receive chemotherapy. After the surgery, the patient developed the symptoms of ileus two or three times within a year; therefore, a synechotomy was performed at 44 years of age. No malignant lesions were found, and the postoperative course was uneventful. However, at 53 years of age, 10 years after the primary tumor resection, the patient was admitted to the emergency room because of abdominal pain, and although the pain disappeared without special therapy, a 4-cm tumor in the lower left abdominal cavity was detected by computed tomography (CT) (fig. 2A), and tumor uptake on a positron emission tomography (PET) scan revealed a maximum standardized uptake value of 9.0 (fig. 2B). Chest CT scan, esophagogastroduodenoscopy, and colonoscopy revealed no malignant lesions. Thus, the diagnosis was a recurrence of ascending colon cancer resected 10 years ago, and a recurrent tumor resection with partial small intestinal resection was performed. This laparotomy revealed only the recurrent tumor detected by CT scan. There were no additional tumors (either primary or recurrent lesions), and the intraoperative cytology of the peritoneal washing showed no evidence of malignancy. Histology of the resected tumor was shown to be moderately differentiated adenocarcinoma ( $\underline{\text { fig. }} 3 \mathrm{~A}$ ) with infiltration into the fat tissue of the omentum and within the wall of the small intestine. Immunohistochemically, adenocarcinoma cells were negative for cytokeratin (CK) 7 (Clone OV-TL 12/30, Dako, Carpinteria, Calif., USA) and strongly positive for CK 20 (Clone Ks20.8, Dako), which is suggestive of colon origin (fig. 3B and C). Characteristically, immunohistochemical expression of CD44 (Clone F10-44-2, Dako), a marker suggestive of colorectal cancer stem cells, labeled a few normal intestinal epithelial cells localized at the bottom of the crypt, which were assumed to be stem cells, and had a scattered distribution in cancer cell membranes (fig. 3D). Vascular endothelial growth factor (VEGF, Clone A-20, Santa Cruz Biotech, Calif., USA) was expressed in endocrine cells in the crypt and diffusely expressed in cancer cells (fig. 3E). Angiogenesis and lymphangiogenesis were detected by CD34 (Clone NU-4A1, Nichirei, Tokyo, Japan) and D2-40 (Clone 
D2-40, Dako), respectively (fig. 3F and G). The margins of the resected tumor were free from invasion. After surgery, the patient received adjuvant chemotherapy consisting of tegafur uracil $\left(300 \mathrm{mg} / \mathrm{m}^{2}\right)$ and calcium folinate $(75 \mathrm{mg})$. Ten months after the first recurrence, CT and PET revealed liver tumors (fig. 2C, D, E and F); therefore, a left lateral segmentectomy and S8 partial hepatectomy without any further adjuvant chemotherapy were performed. Microscopic examination of the liver tumors showed moderately differentiated adenocarcinoma compatible with the first recurrence. Then, 8 months after the second recurrence, CT and PET revealed a $3-\mathrm{cm}$ tumor on the end of the ileum (fig. 2G and H). Prior to surgery for this third recurrence, we treated the patient with 5 cycles of combination chemotherapy consisting of folinic acid, fluorouracil and irinotecan. Tumor resection for the third recurrence including ileocolonic anastomosis, total hysterectomy, and bilateral salpingooophorectomy was performed. Intraoperative chemotherapy with oxaliplatin $\left(60 \mathrm{mg} / \mathrm{m}^{2}\right)$ and fluorouracil $\left(300 \mathrm{mg} / \mathrm{m}^{2}\right)$ and intravenous injection of levofolinate $\left(180 \mathrm{mg} / \mathrm{m}^{2}\right)$ was performed. Microscopic examination of the tumor showed moderately differentiated adenocarcinoma compatible with the first and second recurrence. After surgery, the patient received adjuvant chemotherapy consisting of 5 cycles of combination chemotherapy with folinic acid, fluorouracil and oxaliplatin. The patient is well with no evidence of recurrence 12 months after the third recurrence.

\section{Discussion}

There is no consensus concerning the long-term follow-up of patients who have had colon cancer surgery. Follow-up data suggest that the average disease-free survival time between primary tumor resection and recurrence is one and a half years [7]. Metachronous secondary tumors affect $20-30 \%$ of patients and are usually detected during postoperative follow-up, with a higher incidence during the first 2 years [3]. The present patient was disease-free for 10 years after the primary colon cancer surgery with no evidence of recurrence. Most recurrences of colorectal cancer (89\%-100\%) occur within 5 years [8]. There are a few reports of the development of metastic lesions a remarkably long time after the primary colon cancer surgery [9]. Thus, patient followup for more than 10 years after primary colon cancer surgery is desired.

The mechanisms of tumor dormancy are not clear. It is possible that micrometastases of the primary tumor can remain in a dormant state for a long period [10]. The dormant micrometastases may escape dormancy by increasing their level of angiogenic activity through the disappearance of circulating angiogenesis inhibitors and/or a switch to the angiogenic phenotype by a subset of cells within the micrometastases. In our case, the cancer cells stained positive for VEGF, and angiogenesis (as determined by CD34 staining) and lymphangiogenesis (as determined by D2-40 staining) occurred adjacent to VEGF-positive tumor cell nests at the recurrent site. These results suggest that sufficient levels of nutrient supplementation may have increased cancer cell proliferation such that they escaped from the dormant state. VEGF expressed in endocrine cells located in normal intestinal crypt but not in normal absorptive epithelial cells [11] can be an internal control. High VEGF expression in colon cancer cells is related to low overall survival and a short disease-free survival period [12]. As in the present case, diffuse VEGF positivity may indicate repeated recurrence.

Recently, it has become clear that tumors and hierarchically organized heterogeneous cells contain a cancer stem cell component. The cancer stem cells share many similarities with normal stem cells, such as self-renewing capacity and multilineage differentiation properties [13]. CD44 is a transmembrane glycoprotein that participates in growth, survival, differentiation, and motility [14]. CD44 is a robust 
marker and is of functional importance for colon cancer cells for cancer initiation [14]. In our case, the scattered appearance of CD44-positive cells may suggest continuous tumor growth, metastasis, and cancer recurrence.

At present, there is no published data that outlines the impact of the new systemic therapy regimens when given to patients with intra-abdominal recurrence of colon cancer. Cytoreductive surgery treating macroscopically detectable tumor and intraperitoneal chemotherapy early after surgery to treat residual tumor measuring less than $1 \mathrm{~mm}$ has been proposed [15]. This combined modality has already proved its superiority over standard treatment in patients with colon cancer in a randomized study [16]. However, intraperitoneal chemotherapy modalities vary. The first and most commonly used agent for intraperitoneal chemotherapy in colon cancer is mitomycin $\mathrm{C}$ or fluorouracil [17-19]. However, mitomycin C is not an efficient treatment for metastatic colorectal cancer, and it seems logical to use intraperitoneal oxaliplatin in combination with systemic leucovorin. Elias et al. $[17,20]$ used oxaliplatin for intraperitoneal chemotherapy and reported survival rates of $48.5-54 \%$ at 5 years in patients with carcinomatosis of colorectal origin. In our case, we performed three surgical cytoreductions and administered intraperitoneal fluorouracil and oxaliplatin and systemic levofolinate. The patient is well with no evidence of recurrence more than 12 months after the third recurrence. Our case suggests that colon cancer can metastasize many years after the initial resection and that aggressive local as well as systemic chemotherapy may be required for colon cancer management.

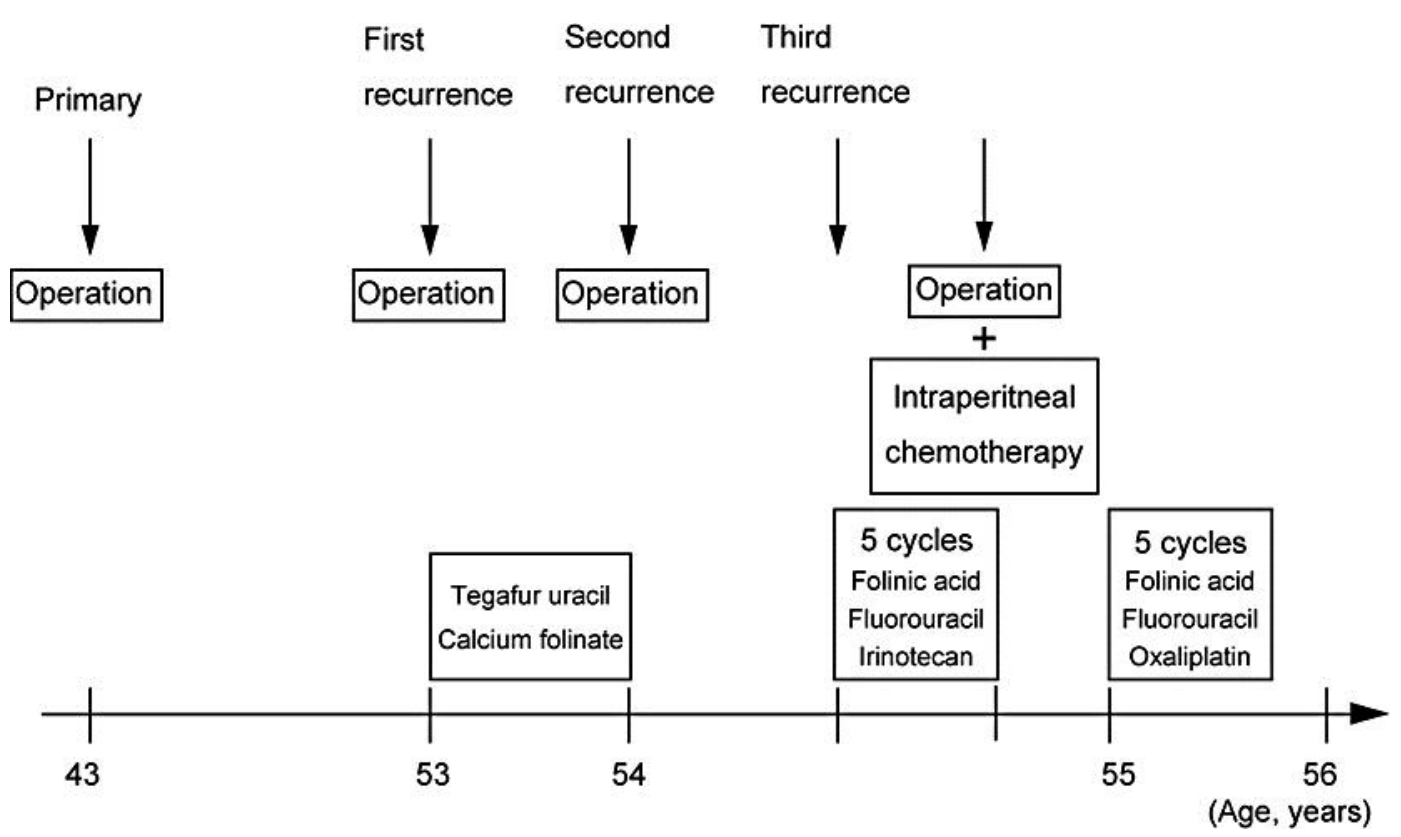

Fig. 1. Schematic representation of the clinical course. 

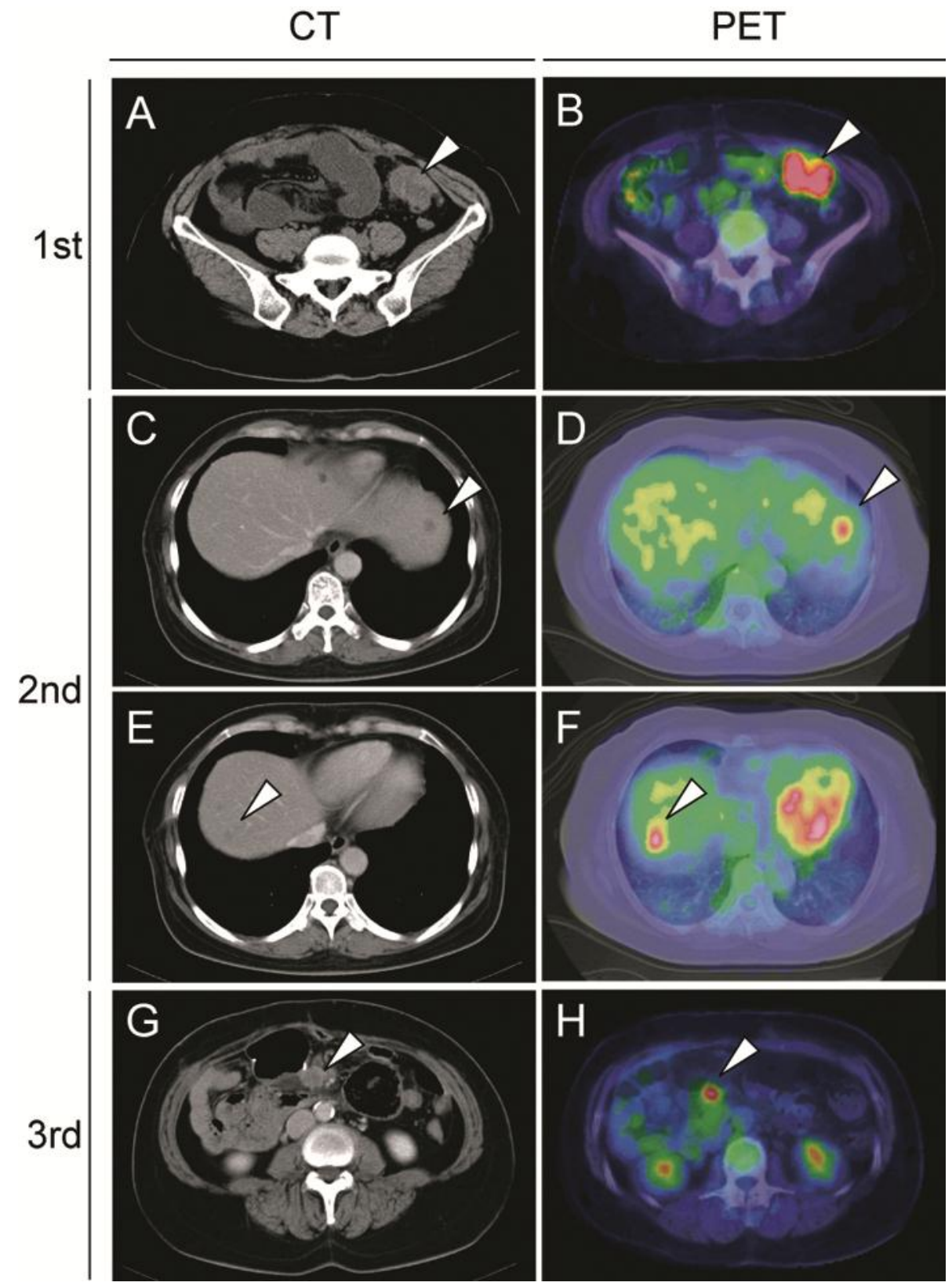

Fig. 2. Abdominal CT scan (A, C, E and G) and PET (B, D, F and H). A and B (arrowhead) show a first recurrence lesion in the left abdomen. $\mathbf{C}, \mathbf{E}, \mathbf{D}$ and $\mathbf{F}$ (arrowhead) show a second recurrence lesion in S3 liver and S8 liver. $\mathbf{G}$ and $\mathbf{H}$ (arrowhead) show a third recurrence lesion in the right abdomen. 

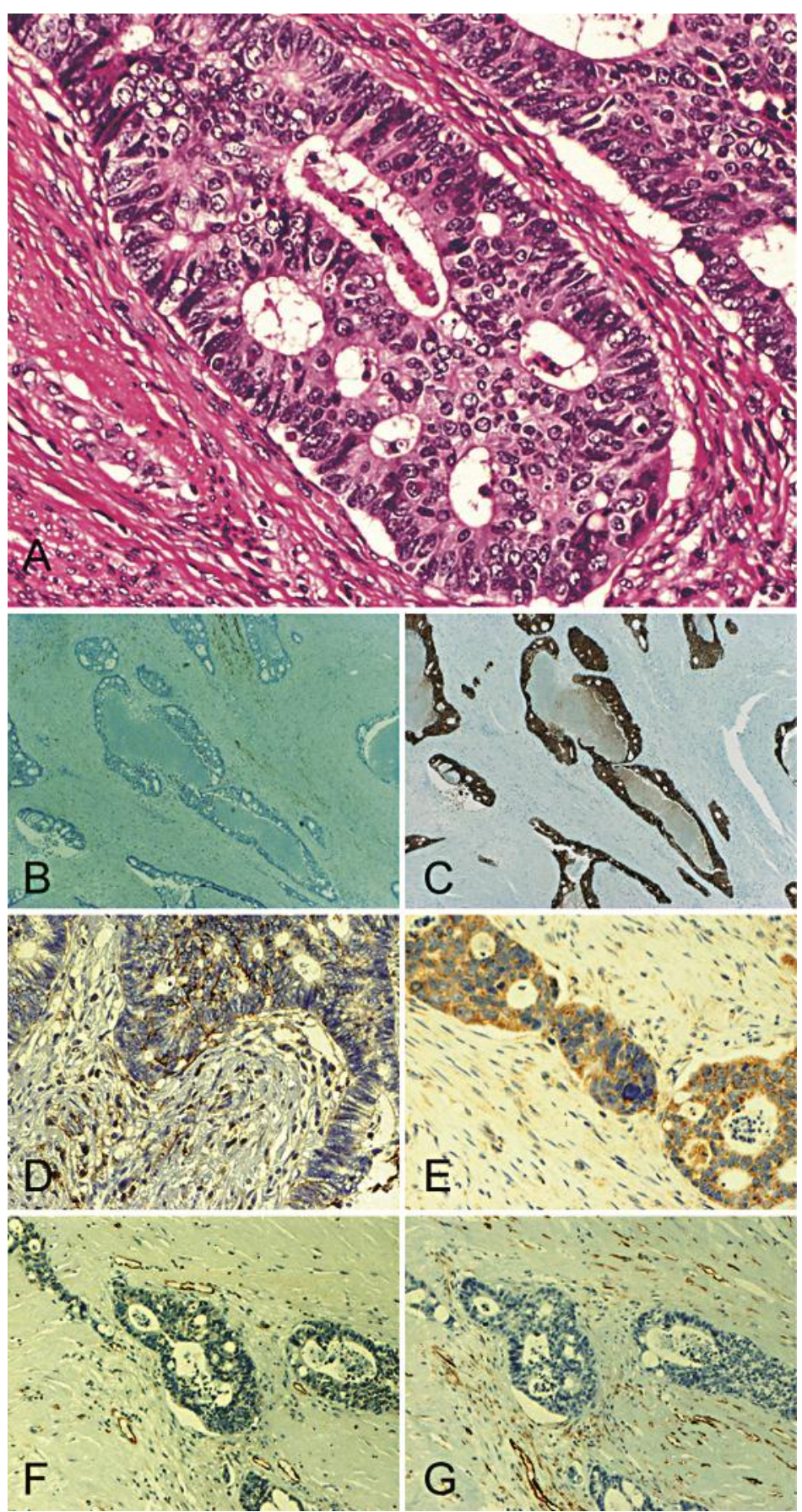

Fig. 3. The histopathologic finding of the first recurrence. A Tumor shows moderately differentiated adenocarcinoma $(\mathrm{HE} \times 400)$. B Tumor was negative for cytokeratin $(\mathrm{CK}) 7(\times 40)$ and positive for CK $20(\times 40)$. The findings are suggestive of a metastatic tumor from primary colon cancer. $\mathbf{D}$ The tumor membrane is sporadically positive for CD44 $(\times 200)$. E The tumor cytoplasm is diffusely positive for vascular endothelial growth factor (VEGF) $(\times 200)$. F Angiogenesis can be seen by CD34-positive cells $(\times 200)$. G Lymphangiogenesis can be seen by D2-40-positive cells $(\times 200)$. Vessel proliferation was seen around the cancer cell foci. 


\section{References}

1 Greenlee RT, Hill-Harmon MB, Murray T, Thun M: Cancer statistics, 2001. CA Cancer J Clin 2001;51:1536.

-2 Hurwitz HI, Fehrenbacher L, Hainsworth JD, Heim W, Berlin J, Holmgren E, Hambleton J, Novotny WF Kabbinavar F: Bevacizumab in combination with fluorouracil and leucovorin: an active regimen for firstline metastatic colorectal cancer. J Clin Oncol 2005;23:3502-3508.

-3 Nuzzo G, Giuliante F, Giovannini I, Tebala GD, Clemente G, Vellone M: Resection of hepatic metastases from colorectal cancer. Hepatogastroenterology 1997;44:751-759.

4 Sugarbaker PH: Managing the peritoneal surface component of gastrointestinal cancer. Part 1. Patterns of dissemination and treatment options. Oncology 2004;18:51-59.

-5 Sugarbaker PH: Managing the peritoneal surface component of gastrointestinal cancer. Part 2. Perioperative intraperitoneal chemotherapy. Oncology 2004;18:207-219.

-6 Glehen O, Mithieux F, Osinsky D, Beaujard AC, Freyer G, Guertsch P, Francois Y, Peyrat P, Panteix G, Vignal J, Gilly FN: Surgery combined with peritonectomy procedures and intraperitoneal chemohyperthermia in abdominal cancers with peritoneal carcinomatosis: a phase II study. J Clin Oncol 2003;21:799-806.

-7 Sato T, Konishi K, Yabushita K, Nojima N, Kimura H, Maeda K, Tsuji M, Miwa A: The time interval between primary colorectal carcinoma resection to occurrence of liver metastases is the most important factor for hepatic resection. Analysis of total course following primary resection of colorectal cancer. Int Surg 1998;83:340-342.

8 Sadahiro S, Suzuki T, Ishikawa K, Nakamura T, Tanaka Y, Masuda T, Mukoyama S, Yasuda S, Tajima T, Makuuchi H, Murayama C: Recurrence patterns after curative resection of colorectal cancer in patients followed for a minimum of ten years. Hepatogastroenterology 2003;50:1362-1366.

-9 Yamaguchi M, Yamanoi A, Igarashi M, Obmori H, Yoshimura H, Tanaka T, Nagasue N: A resected case of liver metastasis from colon cancer that occurred 13 years after a colectomy. Hepatogastroenterology 2008;55:2221-2223.

10 Holmgren L, O’Reilly MS, Folkman J: Dormancy of micrometastases: balanced proliferation and apoptosis in the presence of angiogenesis suppression. Nat Med 1995;1:149-153.

11 Gulubova M, Vlaykova T: Chromogranin A-, serotonin-, synaptophysin- and vascular endothelial growth factor-positive endocrine cells and the prognosis of colorectal cancer: an immunohistochemical and ultrastructural study. J Gastroenterol Hepatol 2008;23:1574-1585.

12 Liang JF, Wang HK, Xiao H, Li N, Cheng CX, Zhao YZ, Ma YB, Gao JZ, Bai RB, Zheng HX: Relationship and prognostic significance of SPARC and VEGF protein expression in colon cancer. J Exp Clin Cancer Res 2010;29:71-90.

13 Clarke MF, Dick JE, Dirks PB, Eaves CJ, Jamieson CH, Jones DL, Visvader J, Weissman IL, Wahl GM: Cancer stem cells-perspectives on current status and future directions: AACR Workshop on cancer stem cells. Cancer Res. 2006;66:9339-9344

14 Du L, Wang H, He L, Zhang J, Ni B, Wang X, Jin H, Cahuzac N, Mehrpour M, Lu Y, Chen Q: CD44 is of functional importance for colorectal cancer stem cells. Clin Cancer Res 2008;14:6751-6760.

-15 Elias DM, Ouellet JF: Intraperitoneal chemohyperthermia: rationale, technique, indications, and results. Surg Oncol Clin N Am 2001;10:915-933.

-16 Verwaal VJ, van Ruth S, de Bree E, van Sloothen GW, van Tinteren H, Boot H, Zoetmulder FA: Randomized trial of cytoreduction and hyperthermic intraperitoneal chemotherapy versus systemic chemotherapy and palliative surgery in patients with peritoneal carcinomatosis of colorectal cancer. J Clin Oncol 2003;21:3737-3743.

17 Elias D, Benizri E, Di Pietrantonio D, Menegon P, Malka D, Raynard B: Comparison of two kinds of intraperitoneal chemotherapy following complete cytoreductive surgery of colorectal peritoneal carcinomatosis. Ann Surg Oncol 2007;14:509-514.

18 Elias D, Blot F, El Otmany A, Antoun S, Lasser P, Boige V, Rougier P, Ducreux M: Curative treatment of peritoneal carcinomatosis arising from colorectal cancer by complete resection and intraperitoneal chemotherapy. Cancer 2001;92:71-76.

19 Sugarbaker PH. Intraperitoneal chemotherapy for treatment and prevention of peritoneal carcinomatosis and sarcomatosis. Dis Colon Rectum 1994;37:S115-S122.

20 Elias D, Raynard B, Farkhondeh F, Goere D, Rouquie D, Ciuchendea R, Pocard M, Ducreux M: Peritoneal carcinomatosis of colorectal origin. Gastroenterol Clin Biol 2006;30:1200-1204. 